\title{
Reconstructive surgery using dual U-shaped rod instrumentation after posterior en bloc sacral hemiresection for metastatic tumor: case report
}

\author{
Seung-Hoon Lim, MD, ${ }^{1}$ Dae-Jean Jo, MD, PhD, ${ }^{2}$ Sung-Min Kim, MD, $\mathrm{PhD},{ }^{2}$ and \\ Young-Jin Lim, MD, PhD'
} ${ }^{1}$ Department of Neurosurgery, Kyung Hee University School of Medicine; and 2Department of Neurosurgery, Spine Center,
Kyung Hee University Hospital at Gangdong, Seoul, Korea

\begin{abstract}
Despite various complications associated with sacrectomy to remove sacral tumors, total or en bloc sacrectomy has been suggested as the most appropriate surgical treatment in such cases. The authors present the case of a 62-year-old male patient with intractable back pain and voiding difficulty whom they treated with posterior en bloc sacral hemiresection followed by reconstruction using dual U-shaped rods. They report that good spinopelvic stability was achieved without complications. The authors conclude that this technique is relatively simple compared with other sacral reconstructive techniques and can prevent complications, including herniation.
\end{abstract}

http://thejns.org/doi/abs/10.3171/2015.2.SPINE14702

KEY WORDS sacral reconstruction; en bloc sacrectomy; dual U-shaped rods; sacral tumor; oncology

$\mathrm{T}$ HE treatment of tumors of the sacrum can be challenging because of the anatomy of the spinopelvic complex and the size of the tumors on presentation. En bloc tumor resection with adequate margins is known as the only effective method of achieving long-term disease control or cure. ${ }^{6,12}$ However, total sacrectomy, or en bloc resection, results in various complications and sometimes requires challenging reconstruction techniques. Many studies have suggested reconstruction techniques after sacrectomy to overcome these problems and improve surgical outcomes. ${ }^{11}$ The purpose of this study is to report our experience with a case of a metastatic tumor of the sacrum that was treated with en bloc sacral hemiresection and reconstruction using dual U-shaped rods instrumentation. In addition, we discuss the advantages of our surgical methods compared with other surgical techniques for patients with sacral tumors.

\section{Case Report}

A 62-year-old male patient was transferred to our clinic for intractable back pain that suggested a sacral tumor. The patient had suffered from lower-back pain 1 year prior that was aggravated 6 months prior to presentation. The pain first presented at night and was sufficiently strong to wake him multiple times. The pain was severe (8-9 on a visual analog scale [VAS]) and intractable, even to morphine treatment. He also suffered from voiding difficulty and frequency.

He had been diagnosed with lung cancer 13 years previously and was determined to have been completely cured after chemotherapy in another clinic. However, no clinical follow-up had been performed since. We consulted a hemato-oncologist, who confirmed that the patient did not need further treatment for lung cancer.

The overall motor power of the patient's right lower extremity was Medical Research Council (MRC) Grade $4 / 5$, but physical examination was difficult due to pain that was aggravated with motion. No deficit of sensation, abnormal deep tendon reflex, or other pathological reflex was observed. Lumbar radiographs and CT showed osteolytic lesions at the sacrum and right ilium (Fig. 1). Lumbar and sacral MR images showed a well-defined lobulated mass at the sacrum and right ilium across the right sacroiliac joint. The mass was about $8.4 \times 7.7 \times 5.8 \mathrm{~cm}$ and extended to the epidural space into the right sacral neural foramen.

ABBREVIATIONS MRC = Medical Research Council; PVA = polyvinyl alcohol; $V A S=$ visual analog scale.

SUBMITTED July 30, 2014. ACCEPTED February 5, 2015.

INCLUDE WHEN CITING Published online July 31, 2015; DOI: 10.3171/2015.2.SPINE14702.

DISCLOSURE The authors report no conflict of interest concerning the materials or methods used in this study or the findings specified in this paper. 

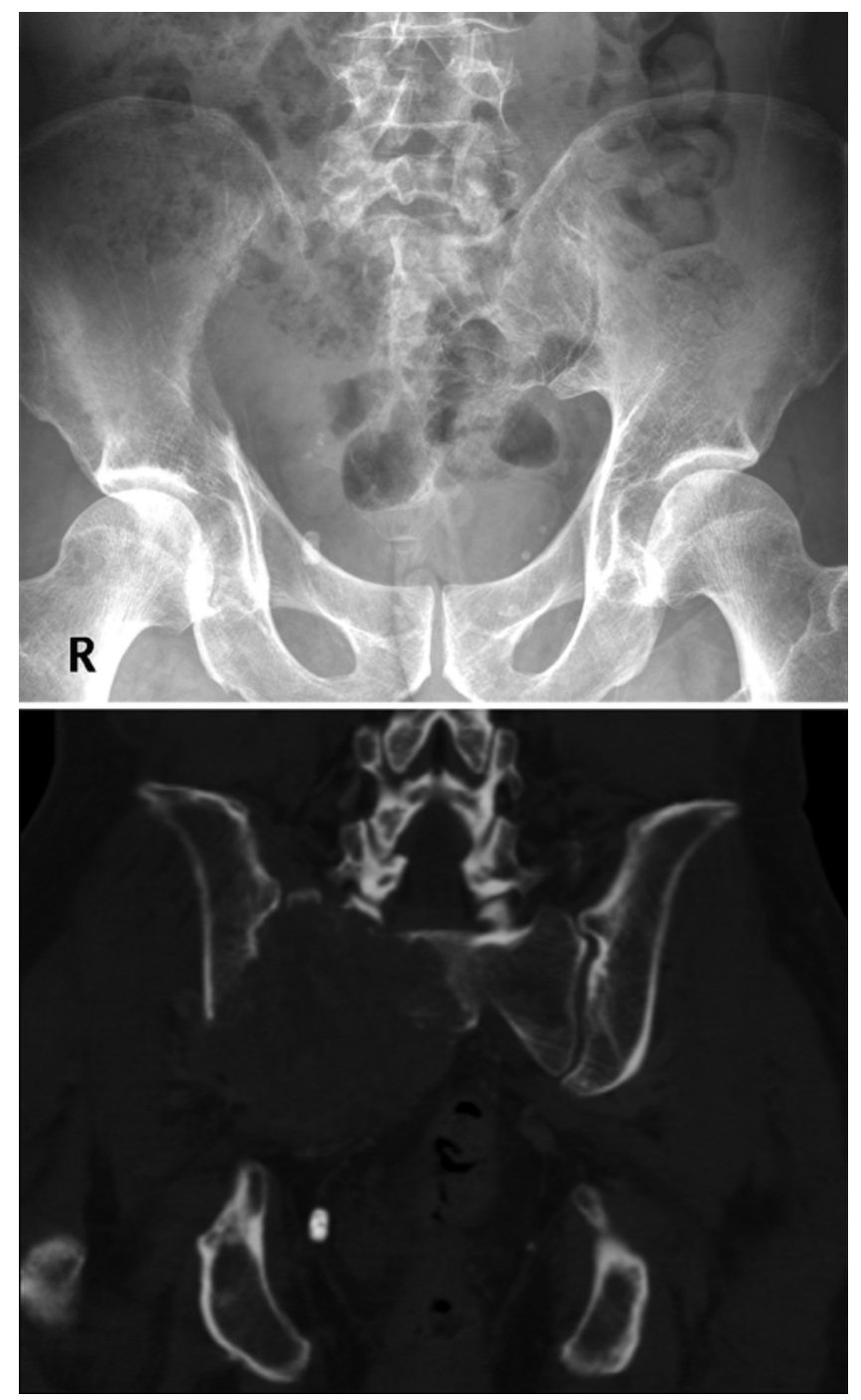

FIG. 1. Anteroposterior pelvic radiograph (upper) and coronal lumbar spine CT image (lower) revealing an osteolytic lesion of the tumor at the sacrum, extending to the right iliac bone.

It showed a heterogeneously low signal intensity on T2weighted images, and mild enhancement with a central nonenhancing area on T1-weighted images obtained after administration of a contrast agent (Fig. 2).

Prior to surgical treatment, tumor embolization was performed using a microcatheter and polyvinyl alcohol (PVA) particles.

Surgery was performed using a posterior-only approach. During the operation, continuous motor evoked potential monitoring was used. A midline incision was carried from L-2 to the level of the coccyx. Because the skin and subcutaneous layer over the coccyx are very thin, the caudal end of the incision was turned slightly to the right side to avoid possible surgical wound problems. Pedicle screws were placed bilaterally at L3-5. Two iliac screws were placed on the left ilium. Because of tumor invasion at the right ilium, 1 iliac screw was placed on the right. The right S-2, S-3, S-4, and S-5 nerve roots were encased by the tumor. The tumor was visualized extend- ing into the L5-S1 intervertebral disc space and S-1 body. Lateral iliac osteotomies were performed using an osteotome, and the L-5 nerve roots were exposed and mobilized from their origin off the thecal sac to the sciatic notch. The right S2-5 nerve roots were ligated and divided. The tumor and nerve roots were exposed sufficiently, and the caudal part of the tumor was dissected by finger dissection. After a total resection of the tumor mass, osteotomy was performed to obtain a safety margin using an osteotome (Fig. 3).

Attention was then turned to reconstruction. As the right sacroiliac joint was invaded by the tumor and removed, reconstruction of the spino-iliac connection was needed. Two rods were bent into a U-shape in the coronal plane to prevent herniation into the defect site; the rods were also bent slightly into an S-shape in the sagittal plane to accommodate lumbar and sacral curvature. After bending the 2 rods, the first U-shaped rod was positioned at the head of pedicle and iliac screws with lateral connectors. Then, the second U-shaped rod was positioned in the lateral connector. Two cross-link connectors were placed. Posterolateral fusion was performed using autologous bone fragments. Postoperative lumbosacral radiography and CT showed good reconstruction (Fig. 4). Histopathological examination revealed squamous cell carcinoma (Fig. 5).

Several days after the surgery, the patient's pain and voiding difficulty were mildly improved, although the right lower-extremity weakness remained unchanged at MRC Grade 4/5. Therefore, we started rehabilitation treatment after a week of bed rest. Clinical follow-up was performed, and no other complications were observed during that period. The last visit was 1 year after surgery. At that time, the patient's pain was reduced (VAS 2-3), but the strength in his right lower extremity remained Grade 4/5. Unfortunately, after 2 years of failed clinical follow-up, we discovered that the patient had died due to other reasons, so no longer-term clinical and radiological outcome data are available.

\section{Discussion}

The indication for radical resection for sacral tumor is malignancy and aggressive benign sacral tumor that is resistant to nonsurgical treatments. ${ }^{3}$ Although total or en bloc sacrectomy is technically difficult and may cause serious structural and neurological damage, it is considered the most appropriate procedure for improving morbidity and mortality for patients with such sacral tumors. ${ }^{5}$ The surgical problems of sacral tumors are associated with their deep location within the pelvis; their relationships with other anatomical structures such as neurovascular systems and pelvic organs; and tumor size that sometimes may extend to iliac bone. ${ }^{6} \mathrm{~A}$ posterior-only approach can be used for lesions that are distal to S-3 or extend proximally from S-3 with no or only minor involvement of the sacroiliac joints. This technique has been reported to allow preservation of roots and reduction of both blood loss and operation time. ${ }^{3}$

Many surgeons have observed various postoperative complications after posterior en bloc sacrectomy, including superficial or deep wound infection, wound dehis- 

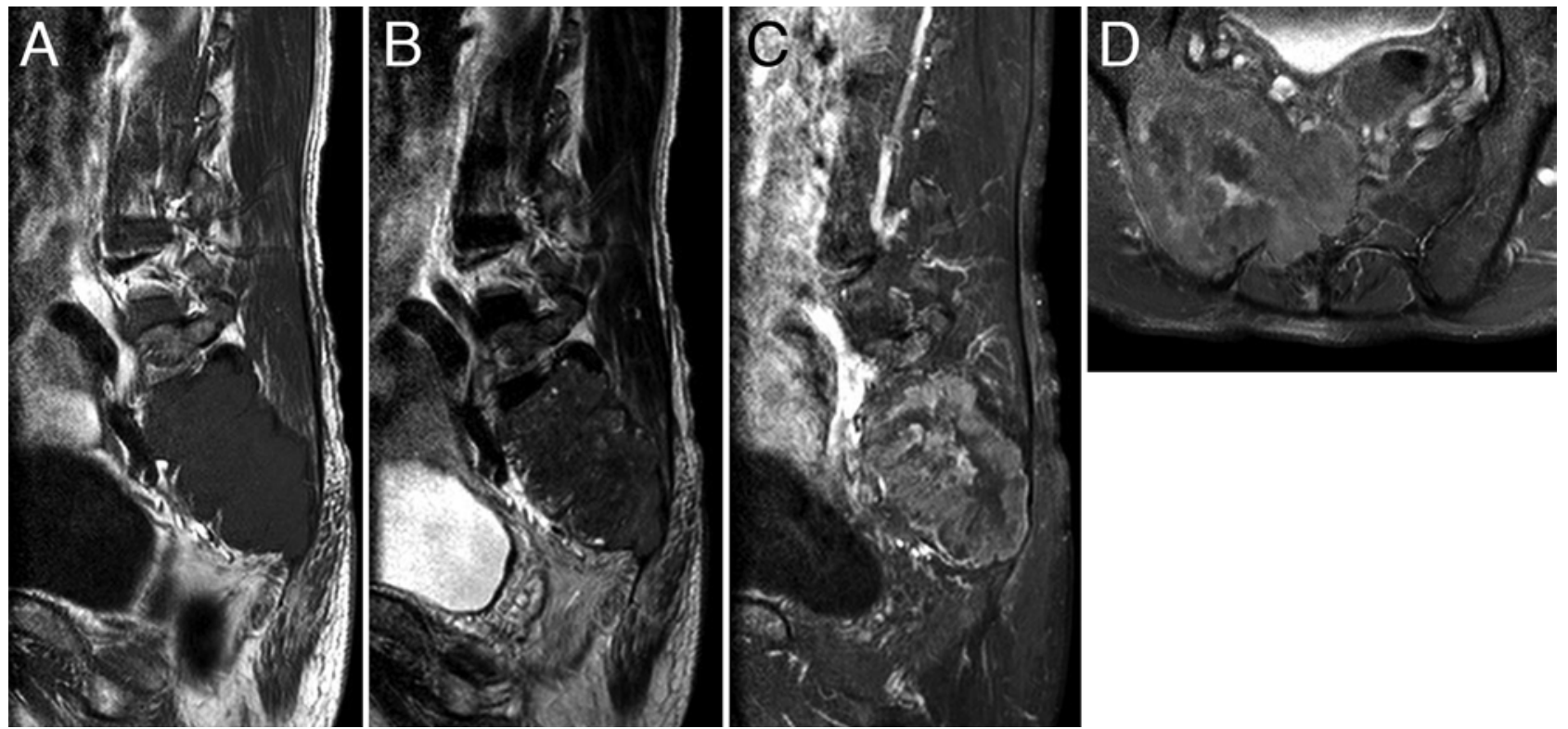

FIG. 2. A and B: Sagittal T1-weighted (A) and T2-weighted (B) MR images showing a mass lesion at the sacrum with heterogeneously low signal intensity. C: Sagittal T1-weighted MR image obtained after administration of contrast medium revealing a mildly enhancing mass lesion with lack of enhancement in the central area. D: Axial T1-weighted MR image obtained after administration of contrast medium showing a well-defined lobulated mass at the sacral body and right ala and right ilium across the right sacroiliac joint.

cence, and pressure necrosis. ${ }^{4,6,13,15,17}$ Because this surgery requires wide dissection due to the removal of a deep and large tumor and the placement of sacroiliac instrumentation, a skin incision is usually performed from the lumbar region to the region of the coccyx, along the median sagittal plane. Occasionally, the incision extends bilaterally to the gluteal region, and has a $\mathrm{Y}$ or triangle shape or other complicated forms., ${ }^{7,12,17}$ These methods can result in wound dehiscence or necrosis that may require a second surgery. ${ }^{15}$ In addition, the skin around the coccyx is very thin and is vulnerable to decubitus ulcer formation. In our case, we performed a straight skin incision from the L-2 level to the coccyx but turned the caudal end of the incision slightly to the right side of the coccyx to prevent wound problems. There were no postoperative wound complications, and the stitches were removed 9 days after surgery. We concluded that a linear skin incision may be a better method to decrease the rate of wound complications, except in the coccygeal area.

Our patient underwent preoperative tumor embolization with PVA. Generally, patients with benign lesions, including aneurysmal bone cysts and giant-cell tumors, respond to embolization with resolution of symptoms and ossification of the lesion. This technique is also used as a primary or adjuvant therapy to reduce tumor size and intraoperative blood loss in patients with malignant or metastatic lesions. ${ }^{18}$ In the present case, because of an uncertain pathological diagnosis and the vascularity of the lesion, we attempted embolization to reduce intraoperative blood loss and improve the surgical outcome.

The focus of our surgical technique is the instrumentation using dual rods. Studies have shown that multiple-rod constructs provide increased stability across osteotomy sites, significantly reducing implant failure and pseudarthrosis compared with a standard single-rod construct. ${ }^{9,14}$
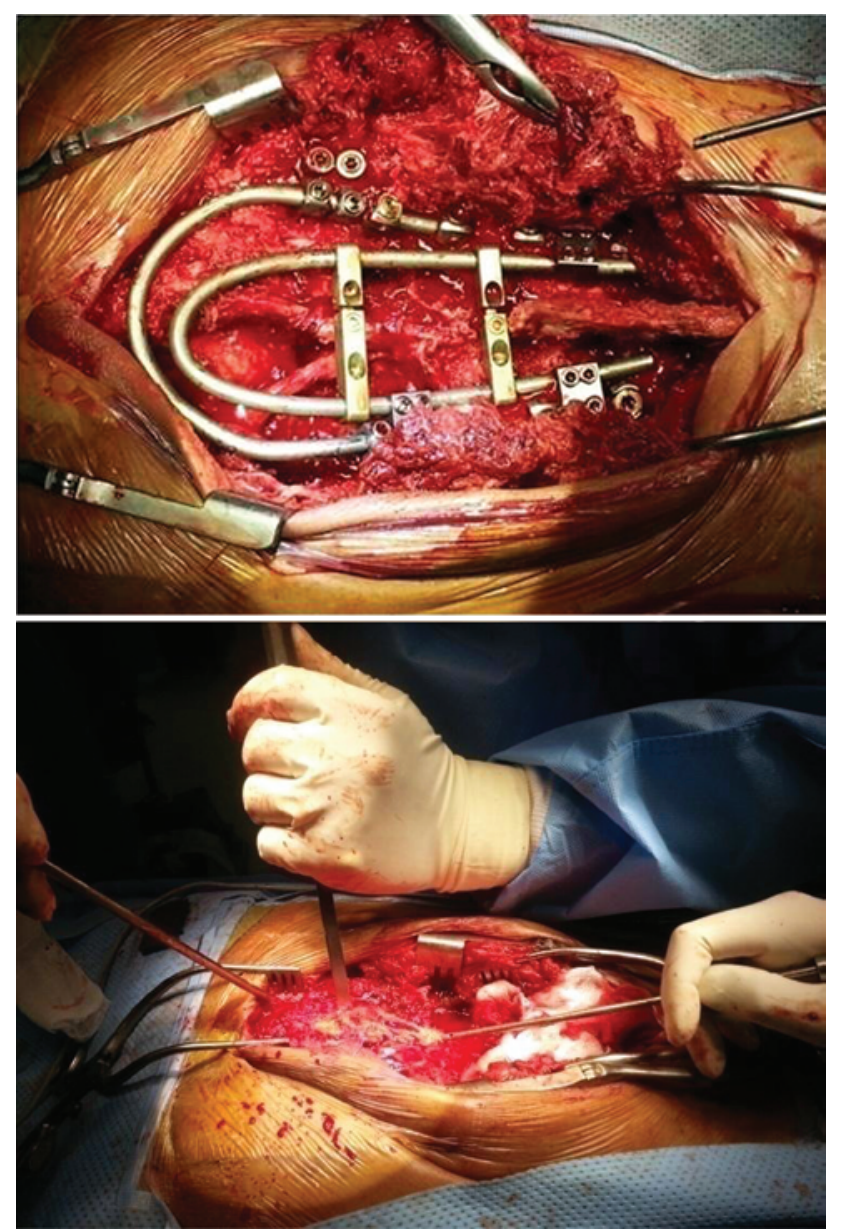

FIG. 3. The operation was performed using a posterior-only method (upper). The sacral tumor and invaded iliac bone were removed using an osteotome (lower). 

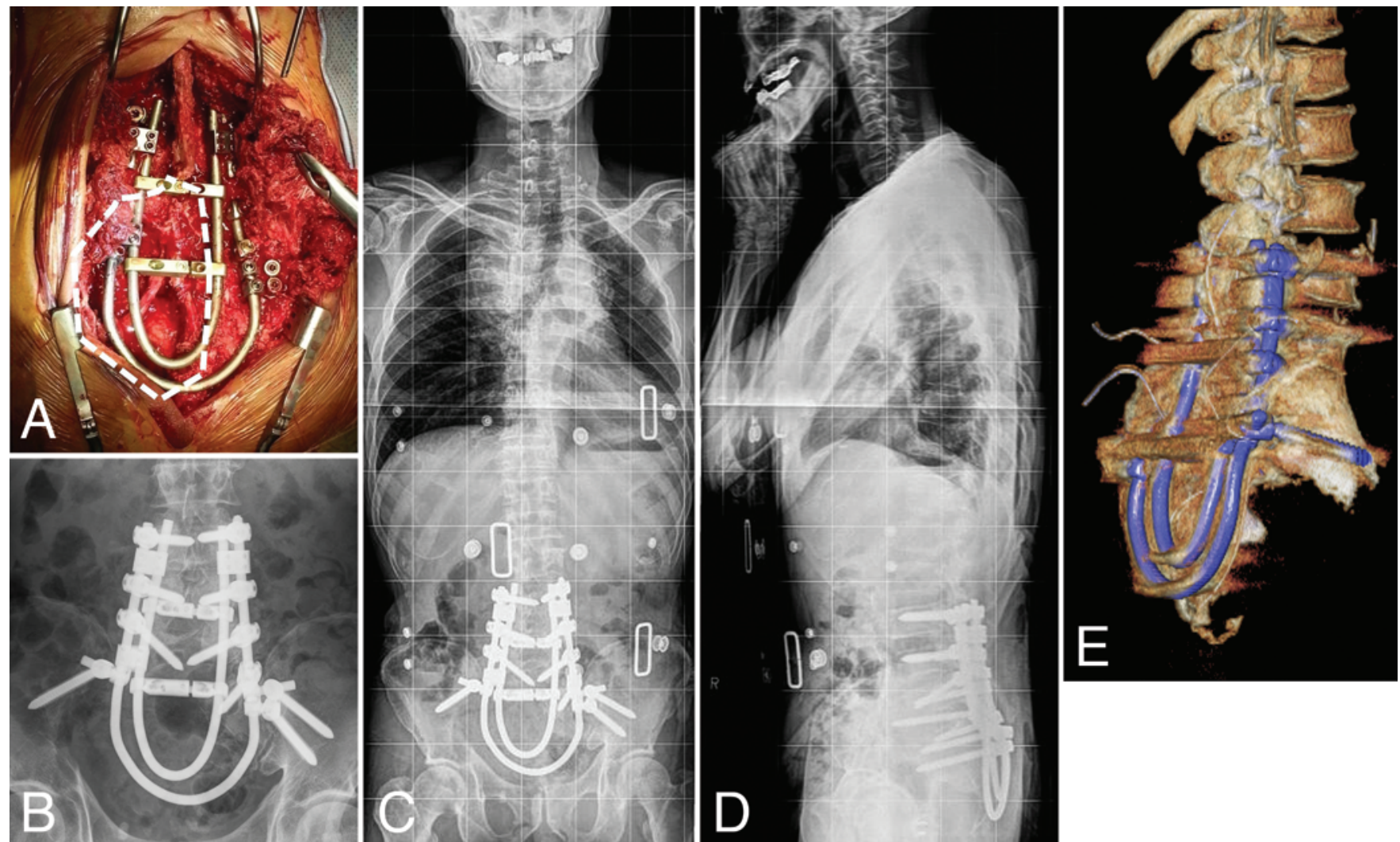

FIG. 4. En bloc hemiresection of the sacrum was performed along the dotted line (A). Postoperative plain radiographs (B-D) and a 3D reconstructed CT image $(E)$ show successful reconstruction and good stability of instrumentation with screws and dual rods, maintaining the lumbar and sacral curvature.

When a second rod is added, the stress on the middle portion of the primary rod is decreased from $50 \%$ to $72 \%$ in flexion, extension, and lateral bending. Sudo et al. reported that dual-rod instrumentation showed good long-term outcomes on radiographic findings and clinical measures at a minimum of 2 years' follow-up. ${ }^{14}$ This method may improve clinical outcomes and prevent pseudarthrosis, rod breakage, loss of correction, and screw pullout by enhancing construct stiffness. ${ }^{16}$ Furthermore, with regard to 3D aspects, we bent the rods into a U-shape in the coronal plane and also curved them slightly in the sagittal plane to maintain lordotic and kyphotic curvatures of the original shape of the spinal column (Fig. 4).

Another advantage of this surgery is the spinopelvic reconstruction technique after en bloc sacrectomy. Various instrumentation techniques have been used for reconstruction after sacrectomy. The method using the Galveston Lrod instrumentation was used for spine deformities in the 1980s. ${ }^{2}$ Gokaslan and colleagues suggested this technique as the optimal reconstructive method after total sacrectomy. ${ }^{8}$ It was reported to provide good spinopelvic stability; however, from a biomechanical viewpoint, weak points have been identified, such as hollows around implants, rod breakage, and implant loosening. Therefore, various techniques that overcome the limitations of the Galveston L-rod technique were proposed, including sacral-rod reconstruction, 4-rod reconstruction, bilateral fibular flaps reconstruction, and improved compound reconstruction. ${ }^{19}$ Although these procedures improved stability and reduced mechanical stress, they could not be used to reconstruct defects following wide resection of the sacrum. Sacral herniation of the abdominal contents is an important and challenging complication that results from defects of the sacrum. The incidence of hernia after sacrectomy is reported to be $1 \%-10 \%,{ }^{1,10}$ while this does not establish a direct relation between the two, sacral herniation remains a problem to be resolved. Many reconstructive options using local flaps, such as from the gluteus or thigh and vertical or transverse rectus abdominis muscle flaps, have been reported. Recently, specific dermal substrates, such as acellular dermal matrix (ADM) or polypropylene mesh, have been used for reconstruction. ${ }^{10}$ In this case, because the sacrum was nearly totally replaced by the dual U-shaped rods, which sufficiently occupied the empty space caused by the defect, we prevented sacral herniation without a specific flap or mesh reconstruction and performed the procedures simply with only a posterior approach.

\section{Conclusions}

Despite various complications associated with sacrectomy to remove sacral tumors, total or en bloc sacrectomy is often suggested as the most appropriate surgical treatment. We found that a reconstructive technique using dual U-shaped rods was useful to prevent herniation of the $a b-$ 


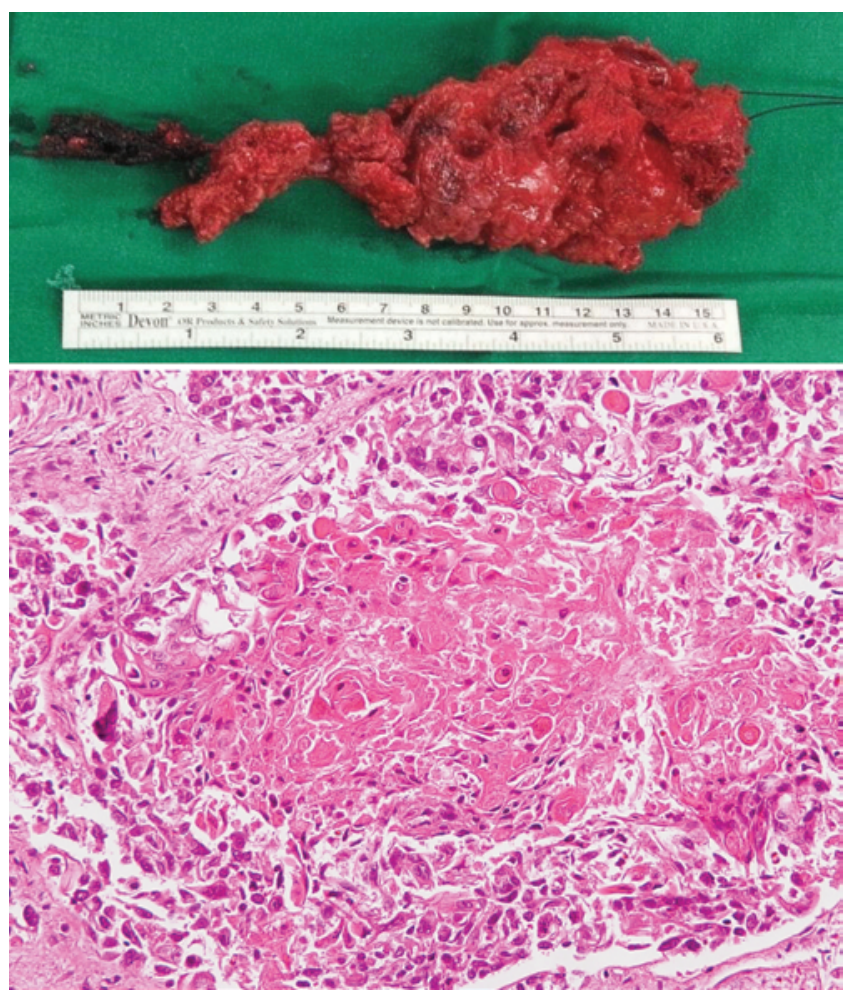

FIG. 5. Gross-total resection of the tumor was achieved (upper), and pathological findings (lower) revealed squamous cell carcinoma with hyperchromatic and pleomorphic nuclei and keratin. $\mathrm{H} \& \mathrm{E}$, original magnification $\times 200$.

dominal contents and to maintain the original lumbar and sacral curves, as well as to improve spinopelvic stability after hemisacrectomy. The advantages of this technique made the procedure simple and prevented complications; however, the surgeon must have the skill to produce the rods in a geometrical configuration that suits the patient.

\section{References}

1. Abhinav K, Shaaban M, Raymond T, Oke T, Gullan R, Montgomery AC: Primary reconstruction of pelvic floor defects following sacrectomy using Permacol graft. Eur J Surg Oncol 35:439-443, 2009

2. Allen BL Jr, Ferguson RL: The Galveston technique of pelvic fixation with L-rod instrumentation of the spine. Spine (Phila Pa 1976) 9:388-394, 1984

3. Angelini A, Ruggieri P: A new surgical technique (modified Osaka technique) of sacral resection by posterior-only approach: description and preliminary results. Spine (Phila Pa 1976) 38:E185-E192, 2013

4. Bederman SS, Shah KN, Hassan JM, Hoang BH, Kiester PD, Bhatia NN: Surgical techniques for spinopelvic reconstruction following total sacrectomy: a systematic review. Eur Spine J 23:305-319, 2014

5. Doita M, Harada T, Iguchi T, Sumi M, Sha H, Yoshiya S, et al: Total sacrectomy and reconstruction for sacral tumors. Spine (Phila Pa 1976) 28:E296-E301, 2003
6. Fourney DR, Rhines LD, Hentschel SJ, Skibber JM, Wolinsky JP, Weber KL, et al: En bloc resection of primary sacral tumors: classification of surgical approaches and outcome. J Neurosurg Spine 3:111-122, 2005

7. Gallia GL, Haque R, Garonzik I, Witham TF, Khavkin YA, Wolinsky JP, et al: Spinal pelvic reconstruction after total sacrectomy for en bloc resection of a giant sacral chordoma. Technical note. J Neurosurg Spine 3:501-506, 2005

8. Gokaslan ZL, Romsdahl MM, Kroll SS, Walsh GL, Gillis TA, Wildrick DM, et al: Total sacrectomy and Galveston Lrod reconstruction for malignant neoplasms. Technical note. J Neurosurg 87:781-787, 1997

9. Hyun SJ, Lenke LG, Kim YC, Koester LA, Blanke KM: Comparison of standard 2-rod constructs to multiple-rod constructs for fixation across 3-column spinal osteotomies. Spine (Phila Pa 1976) 39:1899-1904, 2014

10. Maricevich M, Maricevich R, Chim H, Moran SL, Rose PS, Mardini S: Reconstruction following partial and total sacrectomy defects: an analysis of outcomes and complications. J Plast Reconstr Aesthet Surg 67:1257-1266, 2014

11. Murakami H, Kawahara N, Tomita K, Sakamoto J, Oda J: Biomechanical evaluation of reconstructed lumbosacral spine after total sacrectomy. J Orthop Sci 7:658-664, 2002

12. Newman CB, Keshavarzi S, Aryan HE: En bloc sacrectomy and reconstruction: technique modification for pelvic fixation. Surg Neurol 72:752-756, 2009

13. Schwab JH, Healey JH, Rose P, Casas-Ganem J, Boland PJ: The surgical management of sacral chordomas. Spine (Phila Pa 1976) 34:2700-2704, 2009

14. Sudo H, Ito M, Kaneda K, Shono Y, Abumi K: Long-term outcomes of anterior dual-rod instrumentation for thoracolumbar and lumbar curves in adolescent idiopathic scoliosis: a twelve to twenty-three-year follow-up study. J Bone Joint Surg Am 95:e49, 2013

15. Varga PP, Lazary A: Chordoma of the sacrum: "en bloc" high partial sacrectomy. Eur Spine J 19:1037-1038, 2010

16. Zhang H, Sucato DJ, Pierce WA, Ross D: Novel dual-rod screw for thoracoscopic anterior instrumentation: biomechanical evaluation compared with single-rod and doublescrew/double-rod anterior constructs. Spine (Phila Pa 1976) 34:E183-E188, 2009

17. Zhang HY, Thongtrangan I, Balabhadra RS, Murovic JA, Kim DH: Surgical techniques for total sacrectomy and spinopelvic reconstruction. Neurosurg Focus 15(2):E5, 2003

18. Zhou M, Yang H, Chen K, Wang G, Lu J, Ji Y, et al: Surgical treatment of giant cell tumors of the sacrum and spine combined with pre-operative transarterial embolization. Oncol Lett 6:185-190, 2013

19. Zhu R, Cheng LM, Yu Y, Zander T, Chen B, Rohlmann A: Comparison of four reconstruction methods after total sacrectomy: a finite element study. Clin Biomech (Bristol, Avon) 27:771-776, 2012

\section{Author Contributions}

Conception and design: Jo, SH Lim. Acquisition of data: SH Lim. Reviewed submitted version of manuscript: Kim, YJ Lim. Administrative/technical/material support: Jo.

\section{Correspondence}

Dae-Jean Jo, Department of Neurosurgery, Kyung Hee University Hospital at Gangdong, 892 Dongnam-ro, Gangdong-gu, Seoul 134-727, Korea.email: apuzzo@hanmail.net. 